\title{
The fractional Laplacian as a limiting case of a self-similar spring model and applications to $n$-dimensional anomalous diffusion
}

Manuscript to appear in Fract. Calc. Appl. Anal.

Thomas M. Michelitsch ${ }^{1 *}$ Gérard A. Maugin ${ }^{1}$

Andrzej F. Nowakowski², Franck C. G. A. Nicolleau ${ }^{2}$

Mujibur Rahman ${ }^{3}$

${ }^{1}$ Institut Jean le Rond d'Alembert, CNRS UMR 7190

Université Pierre et Marie Curie (Paris 6)

4 Place Jussieu

75252 Paris Cedex 05

France

\author{
${ }^{2}$ Sheffield Fluid Mechanics Group \\ Department of Mechanical Engineering \\ University of Sheffield \\ Mappin Street, Sheffield S1 3JD \\ United Kingdom \\ 3 General Electric Energy \\ 300 Garlington Road \\ Greenville, SC 29615 \\ USA
}

We dedicate this paper to the memory of our dear friend (and co-author of TMM) Professor Arne Wunderlin (University of Stuttgart, Germany).

July 1, 2013

*Corresponding author, e-mail : michel@lmm.jussieu.fr 
Abstract We analyze the "fractional continuum limit" and its generalization to $n$ dimensions of a self-similar discrete spring model which we introduced recently [21]. Application of Hamilton's (variational) principle determines in rigerous manner a self-similar and as consequence non-local Laplacian operator. In the fractional continuum limit the discrete self-similar Laplacian takes the form of the fractional Laplacian $-(-\Delta)^{\frac{\alpha}{2}}$ with $0<\alpha<2$. We analyse the fundamental link of fractal vibrational features of the discrete self-similar spring model and the smooth regular ones of the corresponding fractional continuum limit model in $n$ dimensions: We find a characteristic scaling law for the density of normal modes $\sim \omega^{\frac{2 n}{\alpha}-1}$ with a positive exponent $\frac{2 n}{\alpha}-1>n-1$ being always greater than $n-1$ characterizing a regular lattice with local interparticle interactions. Furthermore, we study in this setting anomalous diffusion generated by this Laplacian which is the source of Lévi flights in $n$-dimensions. In the limit of "large scaled times" $\sim t / r^{\alpha}>>1$ we show that all distributions exhibit the same asymptotically algebraic decay $\sim t^{-\frac{n}{\alpha}} \rightarrow 0$ independent from the initial distribution and spatial position. This universal scaling depends only on the ratio $n / \alpha$ of the dimension $n$ of the physical space and the Lévi parameter $\alpha$.

Keywords and phrases. Fractional Laplacian, self-similarity, power laws, scale invariance, fractals, Weierstrass-Mandelbrot function, continuum limit, fractional calculus, Fokker Planck equation, anomalous diffusion, Lévi flights, Lévi (stable) distributions, non-locality

\section{Introduction}

Self-similarity (scaling-invariance) can be found in many problems of physics. There is a need for understanding the dynamics that leads to fractal and self-similarity properties in domains of the physics as varied as flow turbulence and complex materials. In "traditional modelling" the continuum is assumed to have a characteristic length scale which determines the wavelengths where wave fields interact with the microstructure. However, there are numerous materials in nature which are constituted by a scale hierarchy of recurrent microstructure which can be conceived in a good approximation as self-similar. This is true for solids and porous media but also in fluid mechanics, or multicomponent and multiphase flows. As a consequence, there are many areas of modelling that could benefit from a better understanding of the mechanisms underlying the dynamics of objects exhibiting self-similarity properties. In fluid mechanics, synthetic turbulence models are one of many examples of tempering with the input spectrum in order to understand better the physics underlying Lagrangian diffusion [26] and such spectra can be traced to the fractal distributions of velocity accumulation points in the synthetic flow. Fractal approaches are developing rapidly in fluid mechanics. Such approaches consist in either experimentally or numerically interfering with the flow, forcing it through self-similar objects (or through numerical forcing) [36, 11, 27].

On the other hand there is a large area of research devoted to the so called Fractional Laplacian and a huge number of references exists, employing this operator in various contexts of physics, e.g. $[33,4,5,10,3,38,40,29,34,17,31,12,13]$. In all these analytical models the fractional Laplacian has been introduced in heuristic manner. In the present paper we show that the fractional Laplacian represents in a unique manner the limiting case of a self-similar lattice Laplacian which we introduced for $1 \mathrm{D}$ in [21]. In that article we introduced self-similar functions in the form of generalized Weierstrass-Mandelbrot functions.

A similar Weierstrass-Mandelbrot type model as in [21] was recently suggested by Nigmatullin and Baleanu [28] to derive functional equations describeing self-similar processes. In that model finite function series with certain scaling properties are analyzed. In this way the self-similarity is only approximate and characteristic length-scales remain present in that model. However, in the limiting case of an infinite series those function series are of the same exactly self-similar WeierstrassMandelbrot function type with fractal features as in our model [21] being the point of departure for the 
present paper. Despite the model [28] is highly useful and advanced to describe fractal and self-similar processes, the "fractional continuum limit" which links the discrete self-similar (fractal) approach with a fractional approach was not considered in that paper.

One aim of the present analysis is hence, to provide a solid physical fundament and justification for the fractional Laplacian based on a simple self-similar "spring model approach". We demonstrate in this paper that the fractional Laplacian is a rigerous consequence (following from Hamilton's variational priciple) defined by the "fractional continuum limit" of the discrete linear spring model of [21] with self-similar and fractal interparticle interactions. In this way we give a natural justification for the appearence of the fractional Laplacian as the smooth field theoretical counterpart of a discrete self-similar (fractal) Laplacian. To this end we evoke the spring model with a self-similar spatial distribution of harmonic interparticle springs [21] and extend it to $n$ dimensions of the physical space. We show that this self-similar Laplacian as well as its generalization to $n$ dimensions can be rigorously obtained by application of Hamilton's variational principle. We generalize the Laplacian to the $n$-dimensional physical space where spatial isotropy of the Laplacian is maintained. It turns out that the $n$-dimensional continuum limit representation of the self-similar Laplacian is up to a positive normalization factor a representation of the $n$-dimensional fractional Laplacian. Throughout the present paper we use synonymously the terms "self-similar Laplacian" in its fractional continuum limit and "fractional Laplacian".

In order to consider a physical application we analyze diffusion processes generated by this Laplacian employed in the Fokker Planck equation. We show that independently of the physical dimension $n$, the underlying anomalous diffusion processes indeed are Lévi flights, which has been demonstrated in the literature for the fractional Laplacian for the spatial dimension $n=2$ [38] and thoroughly analyzed in the survey paper of Metzler and Klafter [20]. An interesting analysis of Lévi flights generated by fractional Laplacians for the mutidimensional space is also presented in [10].

It has been recognized that complex processes exist where the role of fluctuations is by far underestimated and which cannot be described by Gaussian statistics. These random particle motions have much more erratic characteristics as Brownian motions which can be described by Gaussian statistics and normal distributions. This is true for instance for the stock market, where Gaussian statistics fails largely by underestimating fluctuations (risks!). One of the first researchers to recognize this was indeed Mandelbrot $[18,19]$. Among the stochastic motions that are characterized by infinite mean fluctuations are those with Lévi-distributed scale free heavy-tailed jump distributions. A thorough analysis of the jump distribution can be found in the article of Blumenthal et al. [2].

Such erratic motions, allowing long-distance jumps are known in the literature as Lévi flights are the source of anomalous diffusion $[15,20]$ (and references therein). Lévi flights are widely found in nature for instance in the dynamics of bumblebees [14]. There is a huge number of models of Lévi flights by fractional kinetic equations of diffusion and a vast literature devoted to this subject. An excellent overview is provided in [20] (see also the references therein).

However, the subject of the present paper is not the investigation of the properties of Lévi flights at the first place as they are well studied, e.g. [8, 39, 20]. The diffusion problem considered in this paper is only supposed to demonstrate the utility of the self-similar Laplacian approach. Nevertheless we deduce some basic and highly useful properties and relations of Lévi flights in the $n$-dimensional space.

\section{Preliminaries}

It is an interesting question how physical phenomena change when the interparticle interactions become self-similar. For instance when we consider the Poisson equation 


$$
\Delta_{\text {selfsim }} u(\mathbf{r})=\chi(\mathbf{r})
$$

with a self-similar Laplacian operator $\Delta_{\text {selfsim }}$ which is to be specified. To this end we should elaborate first of all the notion of "self-similarity" employed in this paper. The notion of "self-similarity" which is employed in this paper as well as in $[21,22,23,24,25]$ corresponds to the notion "self-similarity at a point' which is commonly used in the mathematical literature [30]. Generally, an object is exactly self-similar in the strict sense if it can be decomposed into parts which are exact rescaled copies of the entire object. In contrast is the notion "self-similarity at a point" where "point" means here a fixed-point of the scaling operation: An object which is self-similar at a point contains a single part which is a re-scaled copy of the entire object and so forth over an infinity of scales [30].

According to this notion of self-similarity we call a function $\Lambda(h)$ self-similar with respect to $h$, i.e. self-similar at point $h=0$, when the scaling relation

$$
\Lambda(N h)=N^{\delta} \Lambda(h)
$$

is fulfilled for a prescribed scaling factor $N>1$ and for any $h>0$. We assume real valued scaling exponents $\delta \in \mathbb{R}$. If a function $\Lambda(h)$ fulfills (2), it follows that (2) remains true if we replace $N \rightarrow N^{s}$ with ( $s \in \mathbb{Z}_{0}$ denotes all positive and negative integers including zero). In other words: if $\Lambda(h)$ is self-similar with respect to $h$ in the sense of relation (2), then there exists a $N>1$ such that the discrete set of rescaling operations $h^{\prime}=h N^{\prime}$ with $N^{\prime}=N^{s}$ with only positive and negative integers $s \in \mathbb{Z}_{0}$ including the zero, satisfy the self-similarity condition (2), namely $\Lambda\left(h N^{\prime}\right)=N^{\prime \delta} \Lambda(h)$. We observe further by putting $h=N^{p+\chi}$ with $p \in \boldsymbol{Z}_{0}$ and $0 \leq \chi<1$ denoting the non-integer part, and by using the property of self-similarity that $\left(N^{p}=h N^{-\chi}\right)$

$$
\Lambda\left(h=N^{p} N^{\chi}\right)=N^{p \delta} \Lambda\left(N^{\chi}\right)=h^{\delta} N^{-\delta \chi} \Lambda\left(N^{\chi}\right)
$$

From this relation we observe that all values of function $\Lambda$ are uniquely determined by its values within $1 \leq N^{\chi}<N$ (as $\left.0 \leq \chi<1\right)$ [23]. It follows that the function $h^{-\delta} \Lambda(h)$ is scaling invariant under $h^{\prime}=N h$ or in other words it is a $\log (N)$-periodic function by conceiving it as function of $\log (h)$ and can be expanded into a log-periodic Fourier series [37, 23]. Log-periodic oscillations appear in many physical processes such as in turbulence as footprint of self-similarity and scaling invariance [28]. This important characteristic feature was probably first discovered by Sornette [37]. The self-similar functions defined in this section can be uniquely expanded in series of log-periodic modes. More details can be found in $[37,23]$.

We notice further that $\Lambda(h)$ scales as $h^{\delta}$, especially when $h \rightarrow 0$. However, in general, a unique limit $h \rightarrow 0$ of $h^{-\delta} \Lambda(h)=N^{-\delta \chi} \Lambda\left(N^{\chi}\right)$ does not exist because of its dependence on $\chi$. Let us assume that a constant $C>0$ exists such that $0 \leq\left|N^{-\delta \chi} \Lambda\left(N^{\chi}\right)\right| \leq C \forall h>0$ then function $\Lambda(h)$ fulfills the inequality

$$
0 \leq|\Lambda(h)| \leq C h^{\delta}
$$

For $0<\delta \leq 1$ relation (4) is the Hoelder condition, e.g. [32]. The function $\Lambda(h)$ is then a Hoelderian function (Hoelder continuous function) being continuous but non-differentiable for $0<\delta<1$ at $h=0$. Hoelderian functions include a wide range of fractal and erratic functions [32]. For $\delta \geq 1$ the function $\Lambda(h)$ is differentiable at $h=0$. Self-similar functions can hence be fractal or non-fractal functions. $[21]$

Generally a self-similar function which fulfills (2) for a prescribed $N$ can be written in the form

$$
\Lambda(h)=\sum_{s=-\infty}^{\infty} N^{-\delta s} f\left(N^{s} h\right)
$$

which converges for sufficiently good functions $f$. Without any loss of generality we can restrict ourselves to $N>1$. The simplest self-similar functions of this type are power-functions $h^{\delta}$. They constitute also the fractional continuum limit of (5) (eq. (9) below). 


\section{The self-similar elastic continuum - the fractional contin- uum limit}

\subsection{One-dimensional case}

In this section we evoke a self-similar Laplacian from a simple linear chain model which was introduced in [21] and its fractional continuum limit we introduced in recent papers [22, 24] for the one-dimensional infinite space. We consider this quasi-continuous chain with self-similar harmonic springs with the Hamiltonian functional [21]

$$
H=\frac{1}{2} \int_{-\infty}^{\infty}\left(\dot{u}^{2}(x, t)+\mathcal{V}(x, t, h)\right) \mathrm{d} x
$$

where $x$ denotes the space- and $t$ the time coordinates. Each spacepoint $x$ indicates a mass point (material point) and the mass density of the system we consider is constant (spatially homogeneous) and we put the mass density equal to $1 . \frac{1}{2} \mathcal{V}(x, h)$ indicates the elastic energy density with

$$
\mathcal{V}(x, h)=\frac{1}{2} \sum_{s=-\infty}^{\infty} N^{-\delta s}\left[\left\{u(x)-u\left(x+h N^{s}\right)\right\}^{2}+\left\{u(x)-u\left(x-h N^{s}\right)\right\}^{2}\right]
$$

which converges for sufficiently regular functions $u(x)$ in the range $0<\delta<2$ and where we assume $h>0$ and $N$ being a prescribed scaling factor. We skip the time coordinate in $u(x)$ whenever the time dependence does not matter. Without loss of generality we can restrict ourselves to $N>1(N \in \mathbb{R})$. The additional factor $1 / 2$ in the elastic energy density compensates double counting of the springs when integrating in (6). (7) describes the harmonic self-similar interaction of a mass point located at $x$ being connected by harmonic springs having spring constants $N^{-\delta s}$ with an ensemble of mass points located at $x \pm h N^{s}$. It is important to note that the elastic energy density does not have any characteristic interaction length scale. The variable $h$ characterizes self-similarity of the elastic energy density, but does not have the physical meaning of a characteristic length. Unlike in the case of a linear chain with only next neighbor interactions, the limit $h \rightarrow 0$ would not localize the interparticle interactions. In (6) and (7) $u$ and $\dot{u}=\frac{\partial}{\partial t} u$ stand for the displacement field (or a generalized field variable) and the velocity field, respectively. (7) has the property of being self-similar with respect to $h$ at point $h=0$, namely

$$
\mathcal{V}(x, N h)=N^{\delta} \mathcal{V}(x, h)
$$

As a starting point for the approach to be developed we evoke the fractional continuum limit of $(7)$ and the resulting equation of motion. For $0<\delta \leq 1$ (7) is a Hoelder continuous function with respect to $h$ in the sense of (4) being for $0<\delta<1$ non-differentiable at $h=0$. If we prescribe in (7) a periodic field $u(x)$ then the elastic energy density is for exponents $0<\delta<1$ a fractal function. We define the fractional continuum limit as $N=1+\zeta(0<\zeta<<1)$ so $\tau=h N^{s}$ becomes a continuous variable and we can write a self-similar function $\Lambda(h)$ which fulfills a self-similarity condition (8) asymptotically as $[21]$

$$
\Lambda(h)=\sum_{s=-\infty}^{\infty} N^{-\delta s} f\left(N^{s} h\right) \approx \frac{h^{\delta}}{\zeta} \int_{0}^{\infty} \frac{f(\tau)}{\tau^{\delta+1}} \mathrm{~d} \tau
$$

having the form of a power function $\Lambda(h)=$ const $h^{\delta}$ where const is independent on $h$. Both the discrete as well as the continuous representation of (9) converge for the same sufficiently good functions (see details in [21]). From (9) follows that in the fractional continuum limit we can write (7) as a functional of the displacement field $u(x)$ in the form 


$$
\mathcal{V}(x, h)=\frac{h^{\delta}}{2 \zeta} \int_{0}^{\infty} \frac{\left\{(u(x)-u(x+\tau))^{2}+(u(x)-u(x-\tau))^{2}\right\}}{\tau^{\delta+1}} \mathrm{~d} \tau
$$

which exists as in the discrete case $(7)$ in the band $0<\delta<2$. Application of Hamilton's principle leads then to the definition of the Laplacian of our system which is then determined by the functional derivative of the total elastic energy $W$ with respect to the field $u$, namely [9]

$$
\Delta_{(\delta, h, \zeta)} u=:-\frac{\delta W}{\delta u}, \quad W=\frac{1}{2} \int \mathcal{V} \mathrm{d} x
$$

The equation of motion ("self-similar wave equation") has then the form [21]

$$
\frac{\partial^{2}}{\partial t^{2}} u(x, t)=\Delta_{(\delta, h, \zeta)} u(x, t)
$$

(11) together with the discrete spring representation (7) defines the discrete form of the self-similar Laplacian [21]

$$
\Delta_{(\delta, N, h)} u(x)=\sum_{s=-\infty}^{\infty} N^{-\delta s}\left\{u\left(x+h N^{s}\right)+u(x-h N)-2 u(x)\right\}
$$

which converges within the same interval as (7), namely $0<\delta<2$ and fulfills the self-similarity condition $\Delta_{(\delta, N, N h)}=N^{\delta} \Delta_{(\delta, N, h)}$. The self-similar Laplacian $\Delta_{(\delta, N, h)}$ defined by (13) is of the type Weierstrass-Mandelbrot function and can be a fractal function if the field $u(x)$ is a periodic function. To demonstrate the link between fractional continuum limit (fractional Laplacian) and fractals represented by the discrete approach, let us evoke the dispersion relation of this Laplacian which is obtained straight-forwardly if one takes into account that this operator is translational invairant with respect to $x$, i.e. has eigenfunctions of the form $e^{i k x}$ which define the dipersion relation (negative eigenvalues of the self-similar Laplacian)

$$
\Delta_{(\delta, N, h)} e^{i k x}=-\omega_{\delta}^{2}(k) e^{i k x}
$$

with the dispersion relation [21]

$$
\omega_{\delta}^{2}(k)=4 \sum_{s=-\infty}^{\infty} N^{-\delta s} \sin ^{2}\left(\frac{k h N^{s}}{2}\right), \quad 0<\delta<2
$$

which is a Weierstrass-Mandelbrot function which again converges for $0<\delta<2$ and is a fractal nowhere differentiable function within $0<\delta<1$ with fractal Hausdorff dimension $D=2-\delta$, and is a regular function witin $1 \leq \delta<2$.

The Weierstrass-Mandelbrot function fulfills also the self-similarity condition with repect to variable $k h$. We further note that (15) is strictly positive for $k \neq 0$ indicating positive definiteness (elastic stability). The self-similar Laplacian is necessarily an elliptic, i.e. non-local and self-adjoint negative (semi-)definite, spatially isotropic operator. ("semi-" because uniform translations are eigenmodes to eigenvalue zero). The fractional continuum limit $N \rightarrow 1$ (relation (9) of the discrete self-similar Laplacian (13) is obtained as

$$
\Delta_{(\delta, h, \zeta)} u(x)=\frac{h^{\delta}}{\zeta} \int_{0}^{\infty} \frac{(u(x-\tau)+u(x+\tau)-2 u(x))}{\tau^{1+\delta}} \mathrm{d} \tau
$$

existing for the same interval $0<\delta<2$. The fractional continuum limit Laplacian (16) can be also obtained from the functional derivative (11) as a consequence of Hamilton's variational principle. The fractional continuum limit $N \rightarrow 1$ can be swaped with application of Hamilton's principle (functional 
derivative (11)). In both cases, in the discrete as well as in the fractional continuum limit the Laplacian is due to its construction self-similar with respect to variable $h$. The dimensional factor $h^{\delta}$ commes into play and maintains the dimension of the discrete self-similar Laplacian (which can be without loss of generality defined as non-dimensional). Performing this fractional limit $N \rightarrow 1$ for the dispersion relation (15) yields a scaling law of the form

$$
\omega_{\delta}^{2}(k)=A_{\delta}|k|^{\delta}, \quad 0<\delta<2
$$

where the strictly positive constant $A_{\delta}>0$ within $0<\delta<2$ is determined below (relations (93) or equivalently by (96) for $n=1$ deduced in the appendix) and has the physical dimension of $h^{\delta}$, i.e. (length) ${ }^{\delta}$. The fractional Laplacian (16) can be written in operator form as

$$
\Delta_{(\delta, h, \zeta)}=-A_{\delta}\left(-\frac{d^{2}}{d x^{2}}\right)^{\frac{\delta}{2}}, \quad 0<\delta<2
$$

with the 1D fractional Laplacian operator $-\left(-\frac{d^{2}}{d x^{2}}\right)^{\frac{\delta}{2}}$ having eigenvalues $-k^{\delta}$ and which approaches the conventional Laplacian for $\delta \rightarrow 2-0$.

It might be sometimes convenient to rewrite (16) in the equivalent form

$$
\Delta_{(\delta, h, \zeta)} u(x)=\frac{h^{\delta}}{\zeta \delta} \frac{d}{d x} \int_{0}^{\infty} \frac{(u(x+\tau)-u(x-\tau))}{\tau^{\delta}} \mathrm{d} \tau, \quad 0<\delta<2
$$

where the range of existence of these relations is $0<\delta<2$. In the entire analysis of this paper we put for any complex number $z=|z| e^{i \varphi}$ the principal value $-\pi<\varphi=\operatorname{Arg}(z) \leq \pi$ for its argument $\varphi$. In the further analysis we will need the $\Gamma$-function (faculty-function) $\Gamma(z)$ which is defined by [1]

$$
\Gamma(\alpha+1)=: \alpha !=\int_{0}^{\infty} e^{-\tau} \tau^{\alpha} \mathrm{d} \tau, \Re(\alpha)>-1
$$

The condition $\Re(\alpha)>-1$ is required for integral (20) to exist. $\Re(Z)$ denotes the real- and $\Im(Z)$ the imaginary part of a complex number $Z$. Using (19) the equation of motion (12) takes the form

$$
\frac{\partial^{2}}{\partial t^{2}} u(x, t)=\frac{\partial}{\partial x} \sigma(x, t)
$$

where $\frac{\partial}{\partial x}$ indicates the traditional partial derivative with respect to $x$ and $\sigma(x, t)$ denote the stress having the form $[24]$

$$
\sigma(x)=\frac{h^{\delta}}{\zeta \delta} \int_{0}^{\infty} \frac{(u(x+\tau)-u(x-\tau))}{\tau^{\delta}} \mathrm{d} \tau, \quad 0<\delta<2
$$

where the integration constant turns out to be zero when integrating the right hand side of (19) with respect to $x$. For further comparison it will be convenient to represent (22) in the equivalent form

$$
\sigma(x)=\frac{h^{\delta}}{2 \zeta \delta} \int_{-\infty}^{\infty} \tau \frac{(u(x+\tau)-u(x-\tau))}{|\tau|^{\delta+1}} \mathrm{~d} \tau, \quad 0<\delta<2
$$

where $\operatorname{sgn}(\tau)=\frac{\tau}{|\tau|}$ maintains the integrand to be an even function with respect to $\tau$.

From the above considerations follows that self-similarity and fractality of the interparticle interactions as introduced by our spring model leads inevitably to non-local fractional field theories. In the elastic framework the material (modulus) functions are convolution kernels. In contrast to the "classical" non-local elasticity theory as outlined by Eringen [7] the self-similar case is characterized by long-range (heavy-tailed) power law kernels decaying critically slowly and without a characteristic length scales. Further details on $1 \mathrm{D}$ cases can be found in $[22,24,25]$. The following section is devoted to the generalization of the approach to $n$-dimensions. 


\section{The fractional continuum limit of self-similar Laplacian in the $n$-dimensional space}

We introduce a generalization of the self-similar Laplacian (16) to the $n$-dimensional space where $n=1,2,3, \ldots$ We define this Laplacian by its action on a scalar field variable $u(\mathbf{x})$. Then we can generalize the one-dimensional case (16) to $n=1,2,3$ dimensions as

$$
\Delta_{(n, \alpha)} u(\mathbf{x})=\frac{h^{\alpha}}{2 \zeta} \int \frac{(u(\mathbf{x}+\mathbf{r})+u(\mathbf{x}-\mathbf{r})-2 u(\mathbf{x}))}{r^{\alpha+n}} \mathrm{~d}^{n} \mathbf{r}
$$

where we have put $\delta-(n-1)=\alpha$. (24) exists for sufficiently smooth fields $u(\mathbf{x})$ converging in the interval $0<\alpha=\delta-(n-1)<2$. Integral (24) is performed over the entire $n$-dimensional physical space $\mathbb{R}^{n}$. The prefactor $2^{-1}$ occurs due to the fact that in (16) a prefactor $2^{-1}$ has to be added to the integrand if we integrate over the entire physical space $\mathbb{R}^{1}$. Expression (24) recovers (16) for $n=1$. The prefactor $h^{\alpha}$ renders the Laplacian defined by (24) non-dimensional. We emphasize again that, as a consequence of self-similarity, the length $h$ does not have the meaning of a characteristic length scale. It represents only a dimensional constant. The fractional continuum limit $N \rightarrow 1$ is up to the dimensional multiplyer $h^{\alpha}$ independent of $h$ (as indicated by the integral on the right hand side of (9)). In some cases also the equivalent representation of (24)

$$
\Delta_{(n, \alpha)} u(\mathbf{x})=\frac{h^{\alpha}}{\zeta} \int \frac{\{u(\mathbf{r})-u(\mathbf{x})\}}{|\mathbf{r}-\mathbf{x}|^{\alpha+n}} \mathrm{~d}^{n} \mathbf{r}, \quad 0<\alpha<2
$$

might be convenient. In the appendix (8.2) we demonstrate that our self-similar Laplacian (24), (25) is up to a strictly positive prefactor coinciding with the fractional Laplacian $-(-\Delta)^{\frac{\alpha}{2}}($ with $\alpha=\delta-(n-1)$ and $0<\alpha<2$ ) known from the literature, e.g. [40] and the references therein.

For the further analysis the following representation of (24) in terms of a divergence of a vector field will be useful

$$
\Delta_{(n, \alpha)} u(\mathbf{x})=\nabla_{\mathbf{x}} \cdot \mathbf{D}(\mathbf{x})
$$

where $\left(\nabla_{\mathbf{x}}\right)_{j}=\frac{\partial}{\partial x_{j}}$ denotes the gradient operator. The vector field $\mathbf{D}$ is determined (up to an unimportant rotational gauge vector field $\mathbf{b}$ with $\nabla \cdot \mathbf{b}=0$ ) by

$$
\mathbf{D}(\mathbf{x})=\frac{h^{\alpha}}{2 \alpha \zeta} \int \frac{\mathbf{r}}{r^{\alpha+n}}\{u(\mathbf{x}+\mathbf{r})-u(\mathbf{x}-\mathbf{r})\} \mathrm{d}^{n} \mathbf{r}, \quad 0<\alpha<2
$$

which recovers for $n=1$ the expression for the stresses (23). The deduction of (27) is performed in the appendix 8.1 by using the Gaussian theorem. Further useful equivalent representations of (27) are given in the appendix. We note that $u$ is here without loss of generality a scalar field and the integrand of (27) is an even function with respect to integration variable $\mathbf{r}$. If we conceive equation (1) as a Poisson equation in an electrodynamic context then the vector field (27) can be conceived as the "dielectric displacement field". Then (1) with (26) defines the self-similar Gauss-law (charge conservation). Starting from this we can set up a potential theory of self-similar fields and apply it for instance to electromagnetism. Some aspects of the theory of self-similar electrostatics are briefly described in [23]. In the appendix 8.1 we have deduced a useful scalar potential (eq. (88)) useful in this context.

\subsection{Dispersion relation and density of normal modes}

In view of the translational symmetry of the Laplacian, we observe that plane-waves $\phi_{k}(\mathbf{r})=e^{i \mathbf{k} \cdot \mathbf{r}}$ are eigenfunctions of the Laplacian (24) where its negative eigenvalues constitute the dispersion relation $\omega_{n, \alpha}^{2}(k)$ which is obtained by the relation 


$$
\begin{array}{cc}
\Delta_{(n, \alpha)} \phi_{k}(\mathbf{r})=-\omega_{n, \alpha}^{2}(k) \phi_{k}(\mathbf{r}) & \\
\omega_{n, \alpha}^{2}(k)=-\frac{h^{\alpha}}{2 \zeta} \int \frac{\left(e^{i \mathbf{k} \cdot \mathbf{r}}+e^{-i \mathbf{k} \cdot \mathbf{r}}-2\right)}{r^{\alpha+n}} \mathrm{~d}^{n} \mathbf{r} & 0<\alpha<2
\end{array}
$$

We observe that the dispersion relation fulfills the scaling property

$$
\omega_{n, \alpha}^{2}(k)=A_{n, \alpha} k^{\alpha}, \quad 0<\alpha<2
$$

depending only on $k=|\mathbf{k}|$ reflecting isotropy of the Laplacian and $A_{n, \alpha}=\omega_{n, \alpha}^{2}(k=1)$ is defined by (29). We observe from (29) that the coefficient $A_{n, \alpha}>0$ is strictly positive indicating "elastic stability". An explicit evaluation of the coefficient $A_{n, \alpha}$ is given in the appendix 8.2. The following observation is noteworthy: In dispersion relation (30) appears always a positive exponent $0<\alpha<2$ being within the interval $(0,2)$ whatever the dimension $n$ of the physical space. From this follows the important property

$$
\omega_{n, \alpha}^{2}(k \rightarrow 0)=0
$$

for any dimension $n$ as a necessary consequence of the translational invariance of the self-similar Laplacian. Translational invariance requires that the $k=0$ mode (uniform translation of the entire "material system") must give a zero contribution to the elastic energy and hence corresponds to eigenvalue zero.

It is now straight-forward to obtain the density of normal modes which we denote by $\mathcal{D}(\omega)$. This quantity is defined such that $\mathcal{D}(\omega) \mathrm{d} \omega$ counts the number of normal modes (per $n$-dimensional unitvolume) with frequencies in the interval $[\omega, \omega+\mathrm{d} \omega]$. We obtain this quantity from the dispersion relation by (e.g. [21] or any textbook of theoretical physics [16])

$$
\mathcal{D}(\omega) \mathrm{d} \omega=\frac{1}{(2 \pi)^{n}} O_{n}(1) k^{n-1} \mathrm{~d} k
$$

where $k=k(\omega)=\frac{\omega^{\frac{2}{\alpha}}}{A^{\frac{1}{\alpha}}}$ represents the inverse dispersion relation and $O_{n}(1)=\frac{2 \pi^{\frac{n}{2}}}{\Gamma\left(\frac{n}{2}\right)}$ denotes the surface of the unit-sphere. Then (32) yields the scaling law

$$
\mathcal{D}(\omega)=\frac{2^{2-n}}{\pi^{\frac{n}{2}} \Gamma\left(\frac{n}{2}\right) \alpha A_{n, \alpha}^{\frac{n}{\alpha}}} \omega^{\frac{2 n}{\alpha}-1}
$$

where the prefactor and exponent $\frac{2 n}{\alpha}-1$ being always positive where $0 \leq n-1<\frac{2 n}{\alpha}-1(0<\alpha<2)$ whatever the dimension $n$ of the physical space. $n-1$ is the exponent of the mode density of the conventional Laplacian having dispersion relation $\omega^{2}(k)=k^{2}$ leading to the density of normal modes in the $n$-dimensional space

$$
\mathcal{D}_{\text {conventional }}(\omega)=\frac{O_{n}(1)}{(2 \pi)^{n}} \omega^{n-1}=\frac{2^{1-n}}{\pi^{\frac{n}{2}} \Gamma\left(\frac{n}{2}\right)} \omega^{n-1}
$$

scaling as $\sim \omega^{n-1}$ and where (33) would assume (34) when we put there the "forbidden" value $\alpha=2$ and $A=1$. We emphasize that in a space of dimension $n$ the exponent of the mode density of the self-similar (fractional) Laplacian is always greater than the exponent of the conventional (localized) Laplacian

$$
\frac{2 n}{\alpha}-1>n-1, \quad 0<\alpha<2
$$


We can qualify inequality (35) as a characteristic footprint of self-similarity. The above power law (33) has due to its positive exponent the property

$$
\mathcal{D}(\omega \rightarrow 0)=0
$$

for any dimension $n$ of the physical space. The positiveness of the exponent reflects the translational invariance of the medium. For $n=1$ the expression obtained in [21] is recovered by (33) with the exponent being $\frac{2}{\delta}-1>0(0<\delta<2)$ and yields

$$
\mathcal{D}_{1}(\omega)=\frac{2}{\pi \delta A_{1, \delta}^{\frac{1}{\delta}}} \omega^{\frac{2}{\delta}-1}
$$

It is quite remarkable that the appearence of the self-similar (fractional) Laplacian gives rise to unusual physical phenomena being qualitatively different from those governed by the conventional Laplacian. Note that diffusion problems formulated with a conventional Laplacian describe traditional Gaussian statistics with finite variances ("Brownian motion"), whereas diffusion problems formulated with self-similar (fractional) Laplacians of the type (24) describe random motions allowing long-range scale-free distributed jumps and leading to stable distributions of the Lévi type, characterized by infinite variances ("Lévi flights")[19]. As an application, we devote the next section to this problem.

\section{$5 \quad$ Anomalous diffusion problem in $n$ dimensions - Lévi flights}

We consider an ensemble of particles of density $\rho(\mathbf{r}, t)$ where $\rho(\mathbf{r}, t) \mathrm{d}^{n} \mathbf{r}$ denotes the number fraction of particles being located at a time $t$ in the volume element $\mathrm{d}^{n} \mathbf{r}$ which is attached to spacepoint $\mathbf{r}$. We refer to this picture as the "diffusion picture". The other picture of this model is a single randomly walking particle where $\rho(\mathbf{r}, t) \mathrm{d}^{n} \mathbf{r}$ then means in this "stochastic picture" the probability to find the particle at time $t>0$ in the volume element $\mathrm{d}^{n} \mathbf{r}$ which is attached to spacepoint $\mathbf{r}$. In what follows we describe a model of the space-time evaluation of the density $\rho$ where both pictures, the diffusion picture and the stochastic picture are possible physical interpretations. In the stochastic picture the present model describes a random walking particle which is walking continuously in time where the jump distance of the particle in any infinitesimal time interval $\delta t$ is distributed according to a power law and isotropic in space. For such stochastic motions Mandelbrot coined the term Lévi flights. We will see that our above introduced self-similar Laplacian operator is the source of exactly this type of stochastic motion in the $n=1,2,3, .$. -dimensional space.

We consider the problem for $t \geq 0$. We are especially interested in the characteristic asymptotic behavior for large times $t$.

The density to be analyzed is normalized for all times $t \geq 0$ according to

$$
\int \rho(\mathbf{r}, t) \mathrm{d}^{n} \mathbf{r}=1
$$

We then define the diffusion problem by the following diffusion equation (in the stochastic picture Fokker Planck equation)

$$
\frac{\partial}{\partial t} \rho(\mathbf{r}, t)=-\mathcal{L}_{n, \alpha} \rho(\mathbf{r}, t)
$$

where $-\mathcal{L}_{n, \alpha}=\Delta_{n, \alpha}$ denotes the Laplacian (24). We further assume a prescribed initial distribution

$$
\rho(\mathbf{r}, t=0)=\rho_{0}(\mathbf{r})
$$

which is also normalized according to (38). We conceive the positive semi-definite operator $\mathcal{L}_{n, \alpha}$ as the diffusion generator where its eigenvalue spectrum is just the dispersion relation (30) with the 
diffusional eigenmodes $\phi_{k}(\mathbf{r})=e^{i \mathbf{k} \cdot \mathbf{r}}$. The density $\rho(\mathbf{r}, t)$ writes in terms of eigenmodes as Fourier transformation

$$
\rho(\mathbf{r}, \mathbf{t})=\frac{1}{(2 \pi)^{n}} \int \hat{\rho}(\mathbf{k}, t) e^{i \mathbf{k} \cdot \mathbf{r}} \mathrm{d}^{n} \mathbf{k}
$$

the Fourier amplitude $\hat{\rho}(\mathbf{k}, t)$ fulfills the evolution equation

$$
\frac{\partial}{\partial t} \hat{\rho}(\mathbf{k}, t)=-\omega_{n, \alpha}^{2}(k) \hat{\rho}(\mathbf{k}, t)
$$

where $\omega_{n, \alpha}^{2}(k)$ is defined by dispersion relation (30). The Fourier amplitudes show an exponential decay in time $\left(A=A_{n, \alpha}>0\right)$

$$
\hat{\rho}(\mathbf{k}, t)=e^{-t A k^{\alpha}} \hat{\rho}_{0}(\mathbf{k})
$$

where we have $0<\alpha<2$ and $\hat{\rho}_{0}(\mathbf{k})$ indicating the Fourier transform of the initial distribution $\rho_{0}(\mathbf{r})$ at $t=0$. Unlike in the case of Gaussian diffusion, the non-locality of Laplacian (24) indicates that non-local particle jumps are admitted which are scale free distributed. We will come back to this important property more closely below.

We can also write the solution of (39) in the form

$$
\rho(\mathbf{r}, t)=e^{-t \mathcal{L}_{n, \alpha}} \rho_{0}(\mathbf{r})
$$

which we will evaluate next. We observe from this representation that the normalization of (44) indeed is maintained at all times $t \geq 0$

$$
\int \rho(\mathbf{r}, t) \mathrm{d}^{n} \mathbf{r}=e^{-t \mathcal{L}_{n, \alpha}} \int \rho_{0}(\mathbf{r}) \mathrm{d}^{n} \mathbf{r}=1 e^{-\mathcal{L}_{n, \alpha} t} 1=1
$$

since $\rho_{0}(\mathbf{r})$ is normalized with $\mathcal{L}_{n, \alpha} 1=0$ so that all powers higher than $m=0$ in the exponential series of the time evolution operator $e^{-\mathcal{L}_{n, \alpha} t} 1$ applied on a constant yield vanishing contributions. This indicates that the total particle number is a conserved quantity. It is further illuminating to consider the diffusion processes more closely: To this end we consider how the density $\rho(\mathbf{r}, t+\delta t)$ evolves from the density $\rho(\mathbf{r}, t)$ where $\delta t$ is an infinitesimal small time interval

$$
\rho(\mathbf{r}, t+\delta t)=e^{-\delta t \mathcal{L}_{n, \alpha}} \rho(\mathbf{r}, t)
$$

where we can put $e^{-\delta t \mathcal{L}_{n, \alpha}} \approx 1-\delta t \mathcal{L}_{n, \alpha}$ and so

$$
\rho(\mathbf{r}, t+\delta t)=\rho(\mathbf{r}, t)-\delta t \mathcal{L}_{n, \alpha} \rho(\mathbf{r}, t)
$$

The operator $\mathcal{L}_{n, \delta}=-\Delta_{n, \alpha}$ is the generator of diffusional processes, generating the infinitesimal transformation of the density from $t$ to $t+\delta t$.

Since $\rho(\mathbf{r}, t) \mathrm{d}^{n} \mathbf{r}$ denotes the particle number fraction being at time $t$ in volume element $\mathrm{d}^{n} \mathbf{r}$ which is attached to the spacepoint $\mathbf{r}$, we can conceive the quantity

$$
\frac{\rho(\mathbf{r}, t+\delta t)-\rho(\mathbf{r}, t)}{\delta t} \sim \frac{\partial}{\partial t} \rho(\mathbf{r}, t)=-\mathcal{L}_{n, \alpha} \rho(\mathbf{r}, t)
$$

as the net balance of the particle number(fraction) departing and arriving in volume element $\mathrm{d}^{n} \mathbf{r}$ during the time interval $\delta t$. In other words (48) measures the number of particles jumping into the volume element minus the number of particles jumping out of the volume element.

The local net balance is due to the non-locality of the operation $\mathcal{L}_{n, \delta} \rho(\mathbf{r}, t)$ depending on all values of $\rho$ at time $t$ in the entire physical space $\mathbb{R}^{n}$ and not (as in the case of Gaussian diffusion) only from 
the $\rho$-values in the local neighborhood. We can express this local balance described by the diffusion equation (39) in terms of a continuity equation

$$
\frac{\partial}{\partial t} \rho(\mathbf{x}, t)=-\nabla_{x} \cdot \mathbf{J}(\mathbf{x}, t)
$$

where we introduced the particle flux density $\mathbf{J}(\mathbf{x})$ which we can write by using (27) in the form

$$
\mathbf{J}(\mathbf{x}, t)=-\frac{h^{\alpha}}{2 \alpha \zeta} \int \frac{\mathbf{r}}{r^{\alpha+n}}\{\rho(\mathbf{x}+\mathbf{r}, t)-\rho(\mathbf{x}-\mathbf{r}, t)\} \mathrm{d}^{n} \mathbf{r}, \quad 0<\alpha<2
$$

which is determined up to an unimportant rotational gauge field (describing closed flux lines which do not change the local density. We observe that equal distributions $\rho(\mathbf{x})=$ const would not cause any particle flux. We can write the flux density also in the equivalent form

$$
\mathbf{J}(\mathbf{x}, t)=\frac{h^{\alpha}}{\alpha \zeta} \int \frac{\mathbf{r}}{r^{\alpha+n}} \rho(\mathbf{x}-\mathbf{r}, t) \mathrm{d}^{n} \mathbf{r}, \quad 0<\alpha<2
$$

where a further equivalent expression is obtained by exchanging $\mathbf{r} \rightarrow-\mathbf{r}$ in (51). For $n=1$ these relations recover the expression found earlier [24]. We can conceive (50) or equivalently (51) as the (non-local) constitutive law connecting particle flux and density replacing the (local) Fick's law of Gaussian diffusion.

Let us consider the particle jump rate into a small volume element $\delta V$ around $\mathbf{r}=0$ due to a localized distribution $Q(\mathbf{x}, t=0)=\delta^{n}(\mathbf{x})$. This localized particle distribution induces at spacepoint of distance $x=|\mathbf{x}|$ instantaneously the flux (due to non-local particle jumps of distance $x$ )

$$
\mathbf{J}(\mathbf{x}, t=0)=\frac{h^{\alpha}}{\alpha \zeta} \frac{\mathbf{x}}{x^{\alpha+n}}
$$

These non-local particle jumps due to the flux (52) cause at spacepoint $\mathbf{x}$ a particle balance which must be positive counting particle jumps from $\mathbf{r}=0$ to $\mathbf{x}$, i.e. jumps over a distance $x$. We obtain with (52)

$$
\frac{\partial}{\partial t} Q(\mathbf{x}, t=0)=-\nabla_{x} \cdot \mathbf{J}(\mathbf{x}, t=0)=\frac{h^{\alpha}}{\zeta} x^{-\alpha-n}>0, \quad \forall \mathbf{x} \neq 0
$$

which is positive and scaling as $\sim x^{-\alpha-n}$ decaying with jump distance $x$ whatever the dimension $n$ since $n<n+\alpha<n+2$ being nonzero in the entire space whatever the jumping distance $x \neq 0$ of the particles. The relation (53) holds everywhere except in the origin $\mathbf{x}=0$. Due to the stochastic spatial isotropy of the particle jumps, integration of (53) over the sphere of radius $R$ gives the total jump rate of all jumps with distance $R$ at $t=0+$. This rate is given for $R \neq 0$ by the first relation

$$
\begin{aligned}
& \operatorname{rate}(R)=\frac{2 \pi^{\frac{n}{2}}}{\Gamma\left(\frac{n}{2}\right)} R^{n-1} \frac{\partial}{\partial t} Q(\mathbf{x}, t=0)=\frac{h^{\alpha}}{\zeta} \frac{2 \pi^{\frac{n}{2}}}{\Gamma\left(\frac{n}{2}\right)} R^{-\alpha-1} \sim R^{-\alpha-1} \\
& \operatorname{Rate}(r>R)=\int_{r=R}^{\infty} \operatorname{rate}(r) \mathrm{d} r=\frac{h^{\alpha}}{\alpha \zeta} \frac{2 \pi^{\frac{n}{2}}}{\Gamma\left(\frac{n}{2}\right)} R^{-\alpha} \sim R^{-\alpha}, 0<\alpha<2
\end{aligned}
$$

where $\frac{2 \pi^{\frac{n}{2}}}{\Gamma\left(\frac{n}{2}\right)} R^{n-1}$ is the surface of the sphere of radius $R$. The second relation gives the rate of jumps over distances greater than $R$ which scales as $R^{-\alpha}$ where $0<\alpha<2$. We can conceive (54) as follows: The probability that a particle which is at $t=0$ located in the origin $\mathbf{r}=0$ undertakes jumps of distance $R$ within the infinitesimal time interval $\delta t$ scales as $R^{-\alpha-1}$. It follows that the total jump 
rate of all jumps over distances greater than $R$ at $t=0+$ decays spatially, scaling with a heavytailed distribution $\sim R^{-\alpha}$ where $0<\alpha<2$ is the band of admissible Lévi-parameter $\alpha$, whatever the dimension $n$ of the physical space. The heavy-tailed power law distribution of jump distances $(54)_{2}$ is nothing but the classical definition of Lèvi flights coined by Mandelbrot [19], indeed proving that diffusion processes generated by the fractional Laplacians in the $n$-dimensional space describe Lévi flights.

A crucial rule plays the space-time representation of the time evolution operator from which we considered the small time regime in (53). This propagator is generally defined by

$$
Q(\mathbf{r}, t)=e^{-t \mathcal{L}_{n, \alpha}} \delta^{n}(\mathbf{r})
$$

where $\delta^{n}(\mathbf{r})$ denotes the $n$-dimensional Dirac's $\delta$-function. In the stochastic picture the interpretation is as follows: The kernel $Q(\mathbf{r}, t)$ describes then the conditional probability density and $Q(\mathbf{r}, t) \mathrm{d}^{n} \mathbf{r}$ denotes the probability to find the particle which was located at $t=0$ in the origin $\mathbf{r}=0$ at time $t$ in the volume element $\mathrm{d}^{n} \mathbf{r}$ attached to the spacepoint $\mathbf{r}$. Correspondingly $Q(\mathbf{r}, t)$ fulfills then the initial condition

$$
Q(\mathbf{r}, t=0)=\delta^{n}(\mathbf{r})
$$

and is hence itself a normalized probability distribution solving (39). We deduce some integral relations thereof in the appendix 8.3. The density (44) can then be represented by the convolution

$$
\rho(\mathbf{r}, t)=\int Q\left(\mathbf{r}-\mathbf{r}^{\prime}, t\right) \rho_{0}\left(\mathbf{r}^{\prime}\right) \mathrm{d}^{n} \mathbf{r}^{\prime}
$$

Taking into account that

$$
\delta^{n}(\mathbf{r})=\frac{1}{(2 \pi)^{n}} \int e^{i \mathbf{k} \cdot \mathbf{r}} \mathrm{d}^{n} \mathbf{k}
$$

together with the property

$$
\left(\mathcal{L}_{n, \alpha}\right)^{m} e^{i \mathbf{k} \cdot \mathbf{r}}=\omega_{n, \alpha}^{2 m}(k) e^{i \mathbf{k} \cdot \mathbf{r}}, \quad m=0,1,2, . . \in \mathbb{N}
$$

where $\omega_{n, \alpha}^{2}(k)$ denotes the dispersion relation (30), we can write for any sufficiently smooth function $f(\xi)$ in the form $f\left(t \mathcal{L}_{n, \alpha}\right) e^{i \mathbf{k} \cdot \mathbf{r}}=f\left(t \omega_{n, \alpha}^{2}(k)\right) e^{i \mathbf{k} \cdot \mathbf{r}}$. By using this property for the exponential operator $e^{-\mathcal{L}_{n, \alpha} t}$ we obtain $Q(\mathbf{r}, t)$ in the form

$$
Q(r, t)=\frac{1}{(2 \pi)^{n}} \int e^{-A_{n, \alpha} k^{\alpha} t} e^{i \mathbf{k} \cdot \mathbf{r}} \mathrm{d}^{n} \mathbf{k}
$$

where $0<\alpha<2$. The kernel is spatially isotropic and depends only on $r=|\mathbf{r}|$ due to the isotropic symmetry of the $\delta$-function. We note that the linear order in $t$ of this Fourier integral coincides with (53) constituting the regime of small times $t$ (appendix 8.3).

Distributions of the form (60) are referred to as Lévi distributions [15, 19]. In contrast to Gaussian distributions, Lévi-distributions exhibit diverging mean fluctuations (all even moments are diverging). This can be directly verified from

$$
<r^{2}>=\left\{\int Q(r, t) r^{2} e^{-i \mathbf{k} \cdot \mathbf{r}} \mathrm{d}^{n} \mathbf{r}\right\}_{k=0}=-\left.\nabla_{k} \cdot \nabla_{k} \tilde{Q}(k, t)\right|_{k=0} \rightarrow \infty
$$

which is fulfilled by $\tilde{Q}(k, t)=e^{-A_{n, \delta} k^{\alpha} t}$ for positive $\alpha$ in the interval $0<\alpha<2$. It is interesting to see that the condition of existence of the Laplacian (24) leads to the same admissible $\alpha$-band $0<\alpha<2$ as the condition of divergence of the variance (61), equivalent with the condition of non-differentiability of $\tilde{Q}(k, t)$ at $k=0$. 
All odd moments are vanishing due to the isotropic symmetry of the distribution $Q(r, t)$. For the further evaluation it is convenient to introduce the following function defined by the surface integral over the unit sphere

$$
G_{n}(\tau)=\frac{1}{(2 \pi)^{n}} \int_{|\hat{k}|=1} \mathrm{~d} \Omega(\hat{k}) e^{\tau \hat{k}_{1}}=\frac{1}{(2 \pi)^{n}} \int \delta(k-1) e^{\tau k_{1}} \mathrm{~d}^{n} \mathbf{k}
$$

where $\hat{k}_{1}$ denotes any Cartesian component of the unit vector $\hat{k}$. We observe due to the spherical symmetry that $G_{n}(\tau)$ contains only even powers in $\tau$, i.e. only the even part $\cosh \tau \hat{k}_{1}$ of the integrand contributes to (62). The surface integral (62) is evaluated explicitly in appendix 8.3 (eq. (114)). The kernel (60) can be further written as

$$
Q(r, t)=r^{-n} P\left(\frac{A_{\alpha, n} t}{r^{\alpha}}\right)
$$

where $P$ is a function of the "scaled time" $\lambda^{\alpha}=\frac{A_{\alpha, n} t}{r^{\alpha}}$ only. In the following we keep in mind that the constant $A_{\alpha, n}$ depends on $n$ and $\alpha=\delta-(n-1)$ and skip these subscripts. The function $P$ takes then the form

$$
P\left(\lambda^{\alpha}\right)=\int_{0}^{\infty} e^{-\lambda^{\alpha} \xi^{\alpha}} G_{n}(i \xi) \xi^{n-1} \mathrm{~d} \xi
$$

To consider large $\lambda^{\alpha}=\frac{A t}{r^{\alpha}}>>1$ we can write by introducing the "fast" variable $u=\lambda \xi$, and we emphasize that $0<\alpha<2$ and hence $\frac{1}{\alpha}$ is a positive exponent, so we can rewrite

$$
P\left(\lambda^{\alpha}\right)=\lambda^{-n} \int_{0}^{\infty} e^{-u^{\alpha}} u^{n-1} G_{n}\left(i \lambda^{-1} u\right) \mathrm{d} u
$$

and hence the kernel $Q=r^{-n} P$ has the form

$$
\begin{gathered}
Q(r, t)=\frac{1}{(A t)^{\frac{n}{\alpha}}} W\left(\frac{r}{(A t)^{\frac{1}{\alpha}}}\right), \quad 0<\alpha<2 \\
W\left(\lambda^{-1}\right)=\int_{0}^{\infty} e^{-u^{\alpha}} u^{n-1} G_{n}\left(i \lambda^{-1} u\right) \mathrm{d} u, \quad \lambda=\frac{(A t)^{\frac{1}{\alpha}}}{r}
\end{gathered}
$$

and the function $G_{n}$ depends only on the dimension $n$ of physical space and is defined by (62). We note that equations (66) with (67) are exact relations. Representation (66) recovers the expression obtained in [24] for one dimension. Generally, except in certain cases to be considered, the integral (67) cannot be obtained in closed form. Despite $\alpha=2$ is a forbidden case in our model (corresponding to Brownian motion described by a diffusion equation with the conventional Laplacian), we can formally consider this case for which (66) with (67) can be evaluated in closed form and lead to a Gaussian distribution

$$
Q_{g}(r, t)=\frac{1}{(4 \pi A t)^{\frac{n}{2}}} e^{-\frac{r^{2}}{4 A t}}
$$

where here $A=A_{2}$ which is not defined by our model as dispersion relation (29) is diverging for $\alpha=2$. Representation (66) is in a sense formally analogous to the Gaussian distribution (68) where the time dependence of the normalization factor of (66) is given by $(A t)^{-\frac{n}{\alpha}}$ and exhibits in the Gaussian case $(\alpha=2)(A t)^{-\frac{n}{2}}$ and leading to $(68)$.

Asymptotic regime $\lambda^{\alpha}=\frac{(A t)}{r^{\alpha}}>>1$ where $\lambda^{\alpha}$ denotes a scaled time: In this regime, relation (67) assumes asymptotically the form 


$$
\begin{gathered}
Q\left(r, A t>>r^{\alpha}\right) \sim \frac{G_{n}(0)}{(A t)^{\frac{n}{\alpha}}}\left(I(n, \alpha)-\frac{r^{2}}{(A t)^{\frac{2}{\alpha}}} I(n+2, \alpha)+. . O\left(\frac{r}{(A t)^{\frac{1}{\alpha}}}\right)^{4}+. .\right) \\
\sim Q(t)=\frac{G_{n}(0)(I(n, \alpha)}{(A t)^{\frac{n}{\alpha}}} \rightarrow 0
\end{gathered}
$$

which decays in the dominant term in time as $t^{-\frac{n}{\alpha}}$ independent on $r$ and where

$$
I(n, \alpha)=\int_{0}^{\infty} e^{-u^{\alpha}} u^{n-1} \mathrm{~d} u
$$

and

$$
G_{n}(0)=\frac{2}{(4 \pi)^{\frac{n}{2}} \Gamma\left(\frac{n}{2}\right)}
$$

The asymptotic relation (69) describes the manner how the distribution approaches the thermodynamic Boltzmannian equal distribution. Since the dominant term in (69) is independent of $r$ and hence does not contain any information about the initial positions of the particles we can conclude that the leading term $Q(t)$ in (69) is universal and holds for any initial distribution $\rho_{0}(\mathbf{r})$. This follows also from the asymptotic relation where we assume that $A t / R^{\alpha}>>1$ and $R>>1$ : The leading contribution to the density in this regime then is (with $Q(\mathbf{r}, t) \sim Q(t)$ )

$$
\rho(\mathbf{r}, t)=\int Q\left(\mathbf{r}-\mathbf{r}^{\prime}, t\right) \rho_{0}\left(\mathbf{r}^{\prime}\right) \mathrm{d}^{n} \mathbf{r}^{\prime} \sim Q(t) \int \rho_{0}\left(\mathbf{r}^{\prime}\right) \mathrm{d}^{n} \mathbf{r}^{\prime}=Q(t)
$$

which leads indeed for any initial distribution $\rho_{0}(\mathbf{r})$ to the identical spatially uniform asymptotics $Q(t) \sim t^{-\frac{n}{\alpha}} \rightarrow 0$ where the exponent depends only on the ratio $\frac{n}{\alpha}$ of the physical dimension $n$ and the Lévi parameter $\alpha$ with the restriction $\frac{n}{2}<\frac{n}{\alpha}<\infty$. If time $t$ exceeds all limits, the system of diffusing particles "forgets" its past approaching all the same thermodynamic attractor $Q(t) \rightarrow 0$. However, since the scaled time $A t / r^{\alpha}$ is for finite $t$ never a large quantity in the entire space as there is always a region where $A t / r^{\alpha}<<1$ is still small and hence (69) is not (yet) valid there. In other words: The spherical region for which the spatially quasi-equal distribution $Q(t)$ of (69) is valid and hence which is close to thermodynamic equilibrium is expanding in time slower than $r(t) \sim(A t)^{\frac{1}{\alpha}}$. The volume of this expanding region scales as $V(t) \sim r^{n}(t) \sim(A t)^{\frac{n}{\alpha}}$. The asymptotic uniform distribution $Q(t) \sim 1 / V(t) \sim(A t)^{-\frac{n}{\alpha}}$ scales as the inverse of the volume $V(t)$ of thermodynamic quasi-equilibrium where $Q(t)$ is the quasi-equal distribution within this region of "quasi-equilibrium". The universal scaling behavior of the type (69) in fractional models of anomalous diffusion was already noted earlier [20].

For all dimensions $n$ of the physical space holds: The algebraic decay of the kernel $Q(r, t) \sim Q(t)$ being independent from $r$ for large scaled times $A t / r^{\alpha}>>1$ is a necessary consequence of Boltzmann's postulate saying that the location of a diffusing particle becomes completely undetermined without any preferred location in the thermodynamic equilibrium indicating the complete "loss of information" about the location of the particle. This corresponds to the thermodynamically necessary condition of approaching maximum (infinite) entropy. We will demonstrate this by the following brief consideration of the entropy which we define here as negative H-function [16]

$$
S(t) \sim-\int Q(r, t) \log Q(r, t) \mathrm{d}^{n} \mathbf{r}
$$

We are especially interested in this quantity in the large times limit $t \rightarrow \infty$. In this regime we can put

$$
S(t) \sim-\log \left\{(A t)^{-\frac{n}{\alpha}}\right\} \int Q(r, t) \mathrm{d}^{n} \mathbf{r} \sim \frac{n}{\alpha} \log (t) \rightarrow \infty
$$


which diverges logarithmically and spatially homogenously in time indicating in the stochastic picture that the state of complete uncertainty about the location of the particle is approached as the global time $t$ tends to infinity. It follows that (74) is universal and independent from the initial distribution $\rho_{0}(\mathbf{r})$. In other words relation (74) expresses the validity of the H-theorem due to Boltzmann which is equivalent to the fact that distributions are broadening in time approaching equal distribution as time tends towards infinity. The divergence of the maximum entropy in (74) is reflecting the fact that the available volume for any particle becomes infinite as $t \rightarrow \infty$ whereas the particle number remains a finite constant. The entropy would approach a finite equilibrium value if for $t \rightarrow \infty$ the volume accessible for the particles would be finite. A finite volume, however would be in contradiction to the self-similarity and as a consequence non-locality of the Laplacian. For a "real" physical system in nature therefore, self-similarity can only be approximatively fulfilled.

Let us briefly consider the one-dimensional case $n=1$ : There we have $\alpha=\delta-(n-1)=\delta$ with

$$
Q_{1}\left(r, A t>>r^{\delta}\right) \sim \frac{I(1, \delta)}{\pi}(A t)^{-\frac{1}{\delta}}, \quad 0<\delta<2
$$

and

$$
I(1, \delta)=\int_{0}^{\infty} e^{-u^{\delta}} \mathrm{d} u
$$

Expressions (75) with (76) is in accordance with the asymptotic relation obtained in [24] for the one-dimensional case. There is one single case remaining, where $Q(r, t)$ can be obtained in closed form: From (67) that this is the case for $\alpha=n$ which can be only fulfilled for $n<2$. Since $n \in \mathbb{N}$ the only case is $n=\delta=1$. We obtain then

$$
G_{1}(i \xi)=\frac{1}{\pi} \cos \xi
$$

and (67) yields

$$
W\left(\lambda^{-1}\right)=\frac{1}{\pi} \int_{0}^{\infty} e^{-u} \cos \left(\lambda^{-1} u\right) \mathrm{d} u=\frac{1}{\pi} \frac{(A t)^{2}}{(A t)^{2}+r^{2}}
$$

The distribution (66) is then obtained as $\left(r=|x|\right.$ and $\left.\lambda=\frac{A t}{|x|}\right)$

$$
Q(r, t)=\frac{1}{A t} W\left(\lambda^{-1}\right)=\frac{1}{\pi} \frac{A t}{(A t)^{2}+x^{2}}
$$

which is known as Cauchy distribution [19] having also the property of diverging even moments. The Cauchy distribution hence is the result of a Lévi flight for a special Lévi parameter $\alpha=\delta=1$. Sometimes this motion is referred to as Cauchy flight. The Cauchy case stands out in the present model and appears uniquely for $n=\delta=1$. The Cauchy distribution behaves for large At $>|x|$ as $Q(t) \sim \frac{1}{\pi}(A t)^{-1}$ in accordance with $(69)$ for $\alpha=n=1\left(\right.$ with $I(1,1)=1$ and $\left.G_{1}(0)=\frac{1}{\pi}\right)$.

\section{Conclusions}

Starting from the self-similar spring model introduced in [21] we have deduced by application of Hamilton's variational principle a self-similar Laplacian operator by a fractional continuum limit for the $n$-dimensional infinite physical space. This operator fulfils all criteria of ellipticity of a good Laplacian: linearity, self-adjointness, isotropic symmetry, negative semi-definiteness, and translational symmetry. The self-similar symmetry renders this Laplacian operator non-local. We proved that this self-similar Laplacian in its fractional continuum limit coincides (up to a strictly positive normalization factor) with the fractional Laplacian $-(-\Delta)^{\frac{\alpha}{2}}$. 
We gave a physical picture and simple basis by means of a self-similar spring model for this Laplacian which follows naturally from a limiting process (the fractional continuum limit) from the spring model. In this way we have demontrated the correspondence of fractal behavior and fractional models. The self-similarity can be conceived as manifestation of an elastic medium with scaling invariant harmonic interparticle interactions. Employing this approach we have deduced the dispersion relation with the density of normal oscillator modes. The density of normal modes in the frequency space fulfills a scaling law $\sim \omega^{\frac{2 n}{\alpha}-1}$ with a characteristic strictly positive exponent being always greater than $n-1$ which would be the exponent due to a conventional Laplacian of the $n$-dimensional space.

As an application of the approach we analyzed a diffusion problem in the $n$-dimensional space defined by a Fokker-Planck equation by employing the self-similar Laplacian (fractional Laplacian). The model describes anomalous diffusion allowing non-local particle jumps where the jump distances are scale free distributed (Lévi flights). The solutions of the Fokker Planck equation are Lévi-stable distributions with characteristic algebraic decay $\sim t^{-\frac{n}{\alpha}}$ in the regime of large scaled times with the Lévi parameter $\alpha$ being in the interval $0<\alpha<2$ whatever the dimension $n$. The present model of anomalous diffusion may have some applications to model physical phenomena dominated by nonBrownian stochastic erratic motions such as for instance in turbulence and chaos. We hope the present approach inspires further work in such directions.

Moreover, the present approach which links fractal features to fractional calculus can be used as a starting point to describe continuous scale free field problems in statics and dynamics. By employing self-similar harmonic models involving higher order differences of the displacement fields, new representations for the fractional Laplacian can be obtained with exponents $\alpha$ exceeding $\alpha=2$. A model of this type is currently under progress.

\section{Acknowledgement}

The present work has been performed under the auspices of the "ERCOFTAC special interest group 42" (SIG42).

\section{Appendix}

\subsection{Derivation of the vector field (27)}

Here we deduce the vector field ("dielectric displacement") D of relation (27). To this end we start with the Laplacian (24)

$$
\Delta_{(n, \alpha)} u(\mathbf{x})=\frac{h^{\alpha}}{2 \zeta} \int \frac{(u(\mathbf{x}+\mathbf{r})+u(\mathbf{x}-\mathbf{r})-2 u(\mathbf{x}))}{r^{\alpha+n}} \mathrm{~d}^{n} \mathbf{r}, \quad 0<\alpha<2
$$

and use the identity

$$
r^{-\alpha-n}=\frac{-1}{\alpha} \nabla_{\mathbf{r}} \cdot\left(r^{-\alpha-n} \mathbf{r}\right)
$$

with $\nabla \cdot(\mathbf{a} b)=b \nabla \cdot \mathbf{a}+\mathbf{a} \cdot \nabla b$. We note that $0<\alpha<2$ is always non-zero. By applying the Gauss theorem for the boundary integral term we get 


$$
\begin{aligned}
& \int_{V(R)} r^{-\alpha-n}(u(\mathbf{x}+\mathbf{r})+u(\mathbf{x}-\mathbf{r})-2 u(\mathbf{x})) \mathrm{d}^{n} \mathbf{r}= \\
& \int_{\partial V(R)} r^{n-1} \mathrm{~d} \Omega(\mathbf{n}) \cdot\left\{\frac{-1}{\alpha} \cdot\left(r^{-(\alpha+n-1)}\right)(u(\mathbf{x}+\mathbf{r})+u(\mathbf{x}-\mathbf{r})-2 u(\mathbf{x}))\right\} \\
& +\frac{1}{\alpha} \int_{V(R)}\left(r^{-\alpha-n} \mathbf{r}\right) \cdot \nabla_{r}(u(\mathbf{x}+\mathbf{r})+u(\mathbf{x}-\mathbf{r})-2 u(\mathbf{x})) \mathrm{d}^{n} \mathbf{r}
\end{aligned}
$$

The boundary integral over $\partial_{V(R)}$ tends versus zero at least as $R^{-\alpha} \rightarrow 0$ and is hence vanishing as $R \rightarrow \infty$ (since $0<\alpha<2)$. When we further use

$\nabla_{r}(u(\mathbf{x}+\mathbf{r})+u(\mathbf{x}-\mathbf{r})-2 u(\mathbf{x}))=\nabla_{\mathbf{x}}(u(\mathbf{x}+\mathbf{r})-u(\mathbf{x}-\mathbf{r}))$

we can write (80) as a divergence

$$
\Delta_{(n, \alpha)} u(\mathbf{x})=\nabla_{\mathbf{x}} \cdot \mathbf{D}
$$

with the vector field $\mathbf{D}$ being determined (up to a unimportant rotational field)

$$
\mathbf{D}(\mathbf{x})=\frac{h^{\alpha}}{2 \zeta \alpha} \int r^{-\alpha-n} \mathbf{r}\{u(\mathbf{x}+\mathbf{r})-u(\mathbf{x}-\mathbf{r})\} \mathrm{d}^{n} \mathbf{r}, \quad 0<\alpha<2
$$

which is relation (27). We observe that the integrand is an even function of $\mathbf{r}$. Taking into account that the volume integral over any odd function of $\mathbf{r}$ is vanishing we can also write

$$
\mathbf{D}(\mathbf{x})=\frac{h^{\alpha}}{\zeta \alpha} \int \frac{\mathbf{r}}{r^{\alpha+n}} u(\mathbf{x}+\mathbf{r}) \mathrm{d}^{n} \mathbf{r}, \quad 0<\alpha<2
$$

or equivalently (by replacing $\mathbf{x}+\mathbf{r} \rightarrow \mathbf{r}$ )

$$
\mathbf{D}(\mathbf{x})=-\frac{h^{\alpha}}{\zeta \alpha} \int \frac{\mathbf{x}-\mathbf{r}}{|\mathbf{x}-\mathbf{r}|^{\alpha+n}} u(\mathbf{r}) \mathrm{d}^{n} \mathbf{r}, \quad 0<\alpha<2
$$

where only the even part of the integrand namely (84)) contributes. All representations (84) and (85) or (86) exist in the band $0<\alpha<2$ just as the Laplacian (80). (85) can be further written in the form of a gradient of a scalar potential

$$
\mathbf{D}(\mathbf{x})=\nabla_{\mathbf{x}} \Phi(\mathbf{x})
$$

where the scalar potential $\Phi(\mathbf{x})$ can be written as

$$
\Phi(\mathbf{x})=\frac{h^{\alpha}}{\alpha(\alpha+n-2) \zeta} \int|\mathbf{x}-\mathbf{r}|^{2-\alpha-n} u(\mathbf{r}) \mathrm{d}^{n} \mathbf{r}, \quad \delta \neq 1, \quad 0<\alpha<2
$$

For $n=1$ this expression recovers those obtained in our previous paper $([21])$. We note that the scalar $\Phi(\mathbf{x})$ is a convolution of the scalar field $u$ with the convolution kernel being the power function ("Riesz potential") $|\mathbf{r}-\mathbf{x}|^{2-\alpha-n}$. The special case of $2-\alpha-n=0(\delta=1)$ appears only for $n=1$. For $n \geq 2$ we have $2-\alpha-n<0$ and hence $R^{2-\alpha-n}$ is vanishing at infinity and singular at $R=0$. Only the case of one dimension $n=1$ with $0<\delta<2$ has for $0<\delta<1$ the "anomaly" that the Riesz potential is diverging at infinity and vanishing in the origin. It follows that

$$
\Delta_{n, \delta} u(\mathbf{x})=\nabla_{\mathbf{x}} \cdot \nabla_{\mathbf{x}} \Phi(\mathbf{x})
$$

where $\nabla_{\mathbf{x}} \cdot \nabla_{\mathbf{x}}$ denotes the conventional Laplacian of the $n$-dimensional space. Relation (89) recovers for $n=1$ the expression we obtained earlier [21]. 
The case $\delta=1$ which can only occur for $n=1$ furthermore again stands out among all the others. This case e.g. lead us to the Cauchy-distribution in section 5. Evaluation of (88) for $n=\delta=1$ yields

$$
\Phi_{\delta=1, n=1}(\mathbf{x})=-\frac{h}{\zeta} \int_{-\infty}^{\infty} \ln (|x-\tau|) u(\tau) \mathrm{d} \tau
$$

which has been already obtained in [21].

\subsection{Determination of $A_{n, \alpha}$}

In this appendix we determine the constant $A_{n, \alpha}=\omega_{n, \alpha}^{2}(k=1)$ occurring in the dispersion relation (30) $\omega_{n, \alpha}^{2}(k)=A_{n, \alpha} k^{\alpha}$. By introducing spherical coordinates $\mathbf{r} \cdot \mathbf{k}=k r n_{1},\left(\mathbf{n} \cdot \mathbf{k}=k n_{1}\right)$ evaluation of (29) yields the representation

$$
\omega^{2}(k=1)_{n, \alpha}=A_{n, \alpha}=\frac{1}{2} J_{\alpha}^{(n)} A_{1, \alpha}
$$

with

$$
A_{1, \alpha}=\omega_{n=1, \delta=\alpha}^{2}(k=1)=\frac{2 h^{\alpha}}{\zeta} \int_{0}^{\infty} \frac{(1-\cos (\tau))}{\tau^{\alpha+1}} \mathrm{~d} \tau, \quad 0<\alpha<2
$$

In view of (91) we can conclude that the dispersion relation of the $n$-dimensional space corresponds to that one in one dimension up to a prefactor $\frac{J_{\alpha}^{(n)}}{2}$ which depends only on the exponent $\alpha$ and dimension $n$ of the physical space. The constant $A_{1, \alpha}$ of (92) is nothing but the dispersion relation for the onedimensional case $\omega_{n=1, \alpha}^{2}(k=1)$ which we already deduced in the one-dimensional model [24]. For the evaluation we refer to that paper. We obtained there

$$
A_{1, \alpha}=\frac{h^{\alpha} \pi}{\zeta \alpha ! \sin \frac{\pi \alpha}{2}}>0, \quad 0<\alpha<2
$$

where this constant is strictly positive and finite in the admitted range $0<\alpha<2$. In (91) occurs a surface integral on the unit-sphere

$$
J_{\alpha}^{(n)}=\int_{|\mathbf{n}|=1} \mathrm{~d} \Omega(\mathbf{n})\left|n_{1}\right|^{\alpha}=\frac{2 \pi^{\frac{n-1}{2}} \Gamma\left(\frac{\alpha+1}{2}\right)}{\Gamma\left(\frac{\alpha+n}{2}\right)}, \quad 0<\alpha<2
$$

and where we have $J_{\alpha}^{(1)}=2$ for $n=1$. The surface integral (94) is straight-forwardly evaluated from the integral $I_{\alpha}^{(n)}=\int e^{-r^{2}}|x|^{\alpha} \mathrm{d}^{n} \mathbf{r}$ where $x=r n_{1}$ and by seperating the surface integration and the radial integration and by decomposing it into a product of $n$ integrals over the Cartesian coordinates. For $\alpha \rightarrow 0+(94)$ approaches the $(n-1)$-dimensional "surface" of the unit-sphere $O(1)=\frac{2 \pi^{\frac{n}{2}}}{\Gamma\left(\frac{n}{2}\right)}$. We obtain then finally for the constant of (91)

$$
A_{n, \alpha}=\frac{h^{\alpha}}{\zeta} \frac{\pi^{\frac{n+1}{2}}}{\Gamma(\alpha+1) \sin \frac{\pi \alpha}{2}} \frac{\Gamma\left(\frac{\alpha+1}{2}\right)}{\Gamma\left(\frac{\alpha+n}{2}\right)}>0, \quad 0<\alpha<2
$$

being positive within the admissible interval $0<\alpha<2$. After some algebra by using the doublication formula together with Euler reflection formulas for $\Gamma$-functions (see Abramovitz \& Stegun [1], page 256 , formulas 6.1 .18 where $2 z=\alpha+1$ and 6.1 .17 with $z=\frac{\alpha}{2}$ ), one finally arrives for the constant (91) at the expression

$$
A_{n, \alpha}=\frac{h^{\alpha}}{\zeta} \frac{\pi^{\frac{n}{2}} \Gamma\left(\frac{\alpha}{2}\right) \Gamma\left(1-\frac{\alpha}{2}\right)}{2^{\alpha} \Gamma\left(1+\frac{\alpha}{2}\right) \Gamma\left(\frac{\alpha+n}{2}\right)}=\frac{h^{\alpha}}{\zeta} \frac{\pi^{\frac{n}{2}} \Gamma\left(1-\frac{\alpha}{2}\right)}{2^{\alpha-1} \alpha \Gamma\left(\frac{\alpha+n}{2}\right)}, \quad 0<\alpha<2
$$


which is in accordance with the (inverse) normalization constant given in the literature, e.g. in [40] (and see the references therein) which occurs by defining the (negative) fractional Laplacian $(-\Delta)^{\frac{\alpha}{2}}$ as the operator with the Fourier transform $k^{\alpha}$ with $0<\alpha<2$. It follows from the scaling behavior of the dispersion relation $\omega^{2}(k)_{n, \alpha}=A_{n, \alpha} k^{\alpha}$, that the fractional Laplacian $-(-\Delta)^{\frac{\alpha}{2}}$ and self-similar Laplacian defined by (24), (25) are linked by the relation

$$
\Delta_{(n, \alpha)}=-A_{n, \alpha}(-\Delta)^{\frac{\alpha}{2}}, \quad 0<\alpha<2
$$

with the strictly postive prefactor $A_{n, \alpha}$ which is determined by (96) for any dimension $n=1,2,3,$. . of the physical space. For $\alpha \rightarrow 2-0$ the fractional Laplacian $(-\Delta)^{\frac{\alpha}{2}}$ defined by (97) with (24), (25) approaches the conventional Laplacian and for $\alpha \rightarrow 0+$ the unity operator. The normalization constant (96) has the physical dimension of $h^{\alpha}$ (i.e. length ${ }^{\alpha}$ ) compensating the dimension of length ${ }^{-\alpha}$ of the fractional Laplacian rendering $\Delta_{(n, \alpha)}$ dimensionless.

\subsection{Some useful integrals}

In this appendix we deduce a rather remarkable relation when we take into account the following identities, namely those of equations (53) and (55) being the linear order in $t$ of relation (60). First we have

$$
\begin{aligned}
& \frac{\partial Q}{\partial t}(r, t=0)=\Delta_{n, \delta} \delta^{n}(\mathbf{r})=-\frac{A_{n, \alpha}}{(2 \pi)^{n}} \int e^{i \mathbf{k} \cdot \mathbf{r}} k^{\alpha} \mathrm{d}^{n} \mathbf{k} \\
& =\frac{h^{\alpha}}{\zeta} \int \frac{\delta^{n}\left(\mathbf{r}-\mathbf{r}^{\prime}\right)}{r^{\prime \alpha+n}} \mathrm{~d}^{n} \mathbf{r}^{\prime}=\frac{h^{\alpha}}{\zeta} r^{-\alpha-n}
\end{aligned}
$$

where the latter integral is obtained by application of the Laplacian on the $\delta$-function for $\mathbf{r} \neq 0$ and yields (53). The Fourier integral can be rewritten for $r \neq 0$

$$
\frac{\partial Q}{\partial t}(r, t=0)=-\frac{A_{n, \alpha}}{r^{\alpha+n}} \int_{0}^{\infty} G_{n}(i \tau) \tau^{\alpha+n-1} \mathrm{~d} \tau
$$

where $G_{n}$ is the surface integral defined in (62) which is evaluated in closed form below. Comparision with the explicit expression (53) yields the integral relation

$$
\int_{0}^{\infty} G_{n}(i \tau) t^{\alpha+n-1} \mathrm{~d} \tau=-\frac{h^{\alpha}}{\zeta A_{n, \alpha}}<0
$$

\subsubsection{Surface integrals}

We choose a Cartesian coordinate system in which the unit vector $\mathbf{n}=\left(n_{1}, . ., n_{n}\right)^{\operatorname{tr}}($ with $\mathbf{n} \cdot \mathbf{n}=1)$ represents a parameterization of the unit-sphere. Let us then evaluate the integral of an integer power of any Cartesian coordinate

$$
\mathcal{A}_{m}^{(n)}=\int_{|\mathbf{n}|=1} \mathrm{~d} \Omega(\mathbf{n}) n_{1}^{m}, \quad m=0,1,2, . . \in \mathbb{N}_{0}
$$

where we integrate over the surface of the unit-sphere. The superscript $(. .)^{(n)}$ indicates the dimension $(n=1,2,3, . \in \mathbb{N})$ of the space. We observe that all integrals of odd powers (odd functions) $\mathcal{A}_{2 m+1}^{(n)}=0$ are vanishing. Only integrals over even powers are non-vanishing and by applying the Gauss-theorem we find the recursion

$$
\mathcal{A}_{2 m}^{(n)}=\frac{(2 m-1)}{(2 m-2+n)} \mathcal{A}_{2 m-2}^{(n)}
$$


where $n$ denotes the dimension of the space $n=1,2,3, \ldots$ This recursion can be applied $m$-times linking $\mathcal{A}_{2 m}^{(n)}$ with $\mathcal{A}_{0}^{(n)}=O_{n}(1)$ which is the surface of the unit sphere. In this way we obtain

$$
\mathcal{A}_{2 m}^{(n)}=\frac{(2 m-1) . . \times 3 \times 1}{(2 m-2+n) . . \times(n+2) \times n} \mathcal{A}_{0}^{(n)}
$$

The products (each containing $m$ factors) in the nominator and the denominator can be written as

$$
(2 m-1) . . \times 3 \times 1=\frac{(2 m) !}{2^{m} m !}=2^{m} \frac{\Gamma\left(m+\frac{1}{2}\right)}{\Gamma\left(\frac{1}{2}\right)}
$$

and

$$
(2 m-2+n) . . \times(n+2) \times n=2^{m} \frac{\left.\left(\frac{n}{2}-1+m\right)\right) !}{\left(\frac{n}{2}-1\right) !}=2^{m} \frac{\Gamma\left(m+\frac{n}{2}\right)}{\Gamma\left(\frac{n}{2}\right)}
$$

So we obtain for (103)

$$
\mathcal{A}_{2 m}^{(n)}=\frac{(2 m) !\left(\frac{n}{2}-1\right) !}{2^{2 m} m !\left(\frac{n}{2}-1+m\right) !} O_{n}(1), \quad m=0,1,2, . . \mathbb{N}
$$

where $O_{n}(1)$ denotes the surface area of the unit-sphere embedded into the $n$-dimensional space [1]

$$
O_{n}(1)=\frac{2 \pi^{\frac{n}{2}}}{\left(\frac{n}{2}-1\right) !}=\frac{2 \pi^{\frac{n}{2}}}{\Gamma\left(\frac{n}{2}\right)}
$$

and hence

$$
\mathcal{A}_{2 m}^{(n)}=\frac{2 \pi^{\frac{n}{2}}(2 m) !}{2^{2 m} m !\left(\frac{n}{2}-1+m\right) !}=2 \pi^{\frac{n}{2}-1} \frac{\Gamma\left(m+\frac{1}{2}\right)}{\Gamma\left(m+\frac{n}{2}\right)}=J_{\alpha=2 m}^{(n)}
$$

coinciding with (94) for $\alpha=2 m$. So we can perform the unit-sphere surface integral of any sufficiently smooth function $f(\tau)$

$$
\begin{gathered}
f\left(\xi n_{1}\right)=\sum_{m=0}^{\infty} \frac{a_{m}}{m !} \xi^{m} n_{1}^{m} \\
g(\xi)=\int_{|\mathbf{n}|=1} \mathrm{~d} \Omega(\mathbf{n}) f\left(\xi n_{1}\right)
\end{gathered}
$$

where only its even part $\frac{f(\tau)+f(-\tau)}{2}$ (even powers) contributes. By using (101)-(108) we obtain for (110) the series

$$
g(\xi)=\sum_{m=0}^{\infty} a_{2 m} \frac{\mathcal{A}_{2 m}^{(n)}}{(2 m) !} \xi^{2 m}
$$

which is uniquely determined by all even derivatives $a_{2 m}$ of $f(\tau)$ at $\tau=0$.

\section{Explicit evaluation of (62)}

With (111) we can evaluate the function $G_{n}(i \xi)$ which is defined by surface integral (62). The integrand (109) is $f\left(\xi n_{1}\right)=\frac{1}{(2 \pi)^{n}} \cos \xi n_{1}$ (or alternatively $f\left(\xi n_{1}\right)=\frac{1}{(2 \pi)^{n}} e^{i \xi n_{1}}$ since any odd function such as $\sin \xi n_{1}$ yields a zero contribution). Then we obtain for $G_{n}(i \xi)$ with (111) and (106) the following series

$$
G_{n}(i \xi)=\frac{2}{(4 \pi)^{\frac{n}{2}}} \sum_{m=0}^{\infty}(-1)^{m} \frac{1}{m ! \Gamma\left(m+\frac{n}{2}\right)} \frac{\xi^{2 m}}{2^{2 m}}
$$


Taking into account the generalized definition of the Bessel function of the first kind ([1] p. 360, 9.1.10.) for any (integer and non-integer) $\nu \in \mathbb{R}$

$$
J_{\nu}(\xi)=\left(\frac{\xi}{2}\right)^{\nu} \sum_{m=0}^{\infty} \frac{1}{m ! \Gamma(m+\nu+1)}\left(\frac{-\xi^{2}}{4}\right)^{m}
$$

So we obtain for (62) the closed form expression

$$
G_{n}(i \xi)=\frac{1}{(2 \pi)^{\frac{n}{2}} \xi^{\frac{n}{2}-1}} J_{\frac{n}{2}-1}(\xi)
$$

Let us now look especially on the physically important cases of dimensions $n=1,2,3$.

(i) $\mathbf{n}=1$

We have with

$$
2^{2 m} m !\left(m-\frac{1}{2}\right) !=(2 m)(2 m-2) . .2 \times(2 m-1)(2 m-3) . .3 \Gamma\left(\frac{1}{2}\right)=(2 m) ! \sqrt{\pi}
$$

and hence so

$$
J_{-\frac{1}{2}}(\xi)=\sqrt{\frac{2}{\xi}} \pi \sum_{m=0}^{\infty}(-1)^{m} \frac{\xi^{2 m}}{(2 m) !}=\sqrt{\frac{2}{\pi \xi}} \cos \xi
$$

so that

$$
G_{n=1}(i \xi)=\frac{1}{\pi} \cos \xi
$$

which we reverify to be correct by its definition $G_{n=1}(\xi)=\frac{1}{2 \pi}\left(e^{i \xi}+e^{-i \xi}\right)$.

(ii) $\mathbf{n}=\mathbf{2}$

Then we have

$$
J_{0}(\xi)=\sum_{m=0}^{\infty}(-1)^{m} \frac{\xi^{2 m}}{2^{2 m} m ! m !}
$$

and

$$
G_{n=2}(i \xi)=\frac{1}{(2 \pi)} J_{0}(\xi)
$$

which we can again reverify directly by the definition of $G_{2}$

$$
G_{2}(i \xi)=\frac{1}{(2 \pi)^{2}} \int_{0}^{2 \pi} e^{i \xi \cos \varphi} \mathrm{d} \varphi
$$

by taking into account the definition of $J_{0}$.

(iii) $\mathbf{n}=3$

Finally we have

$$
\begin{aligned}
& 2^{2 m} m !\left(m+\frac{1}{2}\right) !=(2 m+1)(2 m-1) \times . .3\left(\frac{1}{2}\right) ! \times 2 m(2 m-2) \times . .2 \\
& =(2 m+1) !\left(\frac{1}{2}\right) !=(2 m+1) ! \frac{\sqrt{\pi}}{2}
\end{aligned}
$$


as $\left(\frac{1}{2}\right) !=\frac{1}{2} \Gamma\left(\frac{1}{2}\right)=\frac{\sqrt{\pi}}{2}$ and hence

$$
J_{\frac{1}{2}}(\xi)=\sqrt{\frac{2 \xi}{\pi}} \sum_{m=0}^{\infty}(-1)^{m} \frac{\xi^{2 m}}{(2 m+1) !}=\sqrt{\frac{2}{\pi \xi}} \sin \xi
$$

so that we obtain

$$
G_{n=3}(i \xi)=\frac{1}{2 \pi^{2}} \frac{\sin \xi}{\xi}
$$

which again is directly verifiable by the definition

$$
\begin{aligned}
& G_{3}(i \xi)=\frac{1}{(2 \pi)^{3}} \int_{0}^{2 \pi} \int_{0}^{\pi} e^{i \xi \cos \theta} \sin \theta \mathrm{d} \theta \mathrm{d} \varphi \\
& =\frac{1}{(2 \pi)^{3}} 4 \pi \int_{0}^{1} \cos (\xi u) \mathrm{d} u=\frac{1}{2 \pi^{2}} \frac{\sin \xi}{\xi}
\end{aligned}
$$

\section{References}

[1] M. Abramowitz, I.A. Stegun (Eds.), Handbook of Mathematical Functions with Formulas, Graphs, and Mathematical Tables. New York: Dover (1972). (Chapter 6).

[2] R. M. Blumenthal, R. K. Getoor and D. B. Ray, On the Distribution of First Hits for the Symmetric Stable Processes. Transactions of the American Mathematical Society 99 No 3 (1961), 540-554.

[3] D. Brockmann and L. Hufnagel, Front Propagation in Reaction-Superdiffusion Dynamics: Taming Levy Flights with Fluctuations. Physical Review Lettetters 98 (2007), 178301.

[4] W. Chen, A new definition of the fractional Laplacian. arXiv:cs/0209020 (2002), http://arxiv.org/abs/cs/0209020.

[5] W. Chen, S. Holm, Fractional Laplacian time-space models for linear and nonlinear lossy media exhibiting arbitrary frequency power-law dependency. Journal of the Acoustical Society of America 114 No 5 (2004), 1424-1430.

[6] Z.Q. Chen, P. Kim, R. Song. Heat kernel estimates for Dirichlet fractional Laplacian. Journal of the European Mathematical Society 12 (2010), 1307-1329.

[7] A.C. Eringen, Nonlocal Continuum Field Theories. 2001, Springer New York, Berlin, Heidelberg (2001), ISBN 0-387-95275-6.

[8] W.G. Glöckle, T.F. Nonnenmacher, A fractional calculus Approach to Self Similar Protein Dynamics. Biophysical Journal 68 (1995), 46-53.

[9] H. Goldstein (1980), Classical Mechanics. Second edition, Addition Wesley (1980), 35-69.

[10] A. Hanyga, Multidimensional solutions of spacetime fractional diffusion equations. Proceedings of the Royal Society A 458, No 2018 (2002), 429-450.

[11] D. Hurst, J.C. Vassilicos, Scalings and decay of fractal-generated turbulence. Physics of Fluids 19 (2007), 035103. 
[12] N. Laskin, Fractals and quantum mechanics. Chaos 10, No 4 (2002).

[13] N. Laskin, Fractional Schrodinger equation. Physical Review E 66 (2002), 056108.

[14] F. Lenz, T.C. Ings, L. Chittka, A.V. Chechkin, R. Klages, Spatiotemporal Dynamics of Bumblebees Foraging under Predation Risk. Physical Review Letters 108, (2012), 098103.

[15] P. Lévi, Processus stochastiques et mouvement Brownien. Reprint, Editions Jacques Gabay (1965).

[16] E.M. Lifshitz, L.P. Pitaevskii, Physical Kinetics. Course of Theoretical Physics 10 (Third edition). Pergamon (1981), ISBN 0-08-026480-8.

[17] G. Lu, J. Zhu, An overdetermined problem in Riesz-potential and fractional Laplacian. Nonlinear Analysis 75 (2012), 3036-3048.

[18] B. Mandelbrot, The Fractal Geometry of Nature. W. Freeman \& Co (1982), ISBN 0-716-71186-9.

[19] B. Mandelbrot Fractales, hasard et finance. Champs sciences (1997), ISBN 978-2-0812-2510-7.

[20] R. Metzler, J. Klafter, The random walk's guide to anomalous diffusion: A fractional dynamics approach. Physics Reports 339 (2000), 1-77.

[21] T.M. Michelitsch, G.A. Maugin, F.C.G.A. Nicolleau, A.F. Nowakowski, S. Derogar, Dispersion relations and wave operators in self-similar quasicontinuous linear chains. Physical Review E 80 (2009), 011135.

[22] T.M. Michelitsch, T.M. (2011), The self-similar field and its application to a diffusion problem. Journal of Physics A: Mathematical and Theoretical 44 (2011), 465206.

[23] T.M. Michelitsch, G.A. Maugin, F.C.G.A. Nicolleau, S. Derogar, Wave Propagation in Quasicontinuous Linear Chains with Self-similar Harmonic Interactions: Towards a Fractal Mechnics. Mechanics of Generalized Continua, Advanced Structured Materials, H. Altenbach, G.A. Maugin, V. Erofeev (Eds.), Springer Berlin, Heidelberg, 7 (2011), 231-244.

[24] T.M. Michelitsch, G.A. Maugin, M. Rahman, S. Derogar, A.F. Nowakowski, F.C.G.A. Nicolleau, A continuum theory for one-dimensional self-similar elasticity and applications to wave propagation and diffusion. European Journal of Applied Mathematics 23 (2012), 709735.

[25] T.M. Michelitsch, G.A. Maugin, M. Rahman, S. Derogar, A.F. Nowakowski, F.C.G.A. Nicolleau, An approach to generalized one-dimensional self-similar elasticity. International Journal of Engineering Science 61 (2012), 103111.

[26] F.C.G.A. Nicolleau, C. Cambon, J.-M. Redondo, J.C. Vassilicos, M. Reeks and A.F. Nowakowski (Eds.) (2011), New approaches in modelling multiphase flows and dispersion in turbulence, fractal methods and synthetic turbulence Springer Science, 18, Ercoftac Series (2011), ISBN 978-94-007-2505-8.

[27] F.C.G.A. Nicolleau, S.M. Salim, A.F. Nowakowski, Experimental study of a turbulent pipe flow through a fractal plate. Journal of Turbulence 12 No 44 (2011), 1-20.

[28] R.R. Nigmatullin, D. Baleanu, The derivation of the generalized functional equations describeing self-similar processes. Fractional Calculus \& Applied Analysis 15, No 4 (2012), 718-740.

[29] M.D. Ortiguera, Riesz potential operations and inverses via fractional centred derivatives. International Journal of Mathematics and Mathematical Sciences, (2006), 48391. 
[30] H.O. Peitgen, H. Jürgens, D. Saupe, Fractals for the Classroom: Part 1: Introduction to Fractals and Chaos. Springer (1991), ISBN-10: 9780387970417.

[31] M. Putinar, A renormalized Riesz potential and applications: Advances in Constructive Approximation, Nashboro Press, Brentwood, TN. (2004), 1-33, ISBN 0-9728482-x-x.

[32] H. Qing, L. Fanghua, Elliptic Partial Differential Equations. New York, Courant Institute of Mathematical Sciences (1997), ISBN 0-9658703-0-8.

[33] M. Riesz, L'intégrale de Riemann-Liouville et le problème de Cauchy. Acta Mathematica 81, No 1 (1949), 1-222.

[34] W. Reichel, Characterization of balls by Riesz-potentials. Annali di Matematica pura ed applicata 188, No 2 (2009), 235-245.

[35] S. Samko, A. Kilbas and O. Marichev, Fractional Integrals and Derivatives: Theory and Applications. Gordon and Breach, London (1993).

[36] R.E. Seoud, J.C. Vassilicos, Dissipation and decay of fractal-generated turbulence. Phys. Fluids 19, No 10 (2007), 105-108.

[37] D. Sornette, Discrete scale invariance and complex dimensions. Physics Reports 297 (1998), 239270.

[38] N. Stollenwerk, J.P. Boto, Fractional calculus and Levy flights: modelling spatial epidemic spreading. Proceedings of 9th Conference on Computational and Mathematical Methods in Science and Engineering, CMMSE 2009 J. V. Aguiar et al. (Eds.), Salamanca (2009),177188.

[39] H.G. Sun, W. Chen, C. Li, Y.Q. Chen, Fractional differential models for anomalous diffusion. Physica A 389 (2010), 2719-2724.

[40] J. L. Vazquez, Nonlinear Diffusion with Fractional Laplacian Operators: Nonlinear partial differential equations: the Abel Symposium 2010; Holden, Helge \& Karlsen, Kenneth H. (Eds.), Springer (2012), 271-298. 